\begin{tabular}{|c|l|}
\hline Title & Integrable four-vortex motion on sphere with zero moment of vorticity \\
\hline Author(s) & Sakajo, Takashi \\
\hline Citation & $\begin{array}{l}\text { Physics of Fluids, 1911),017109 } \\
\text { https://doi.org/10.1063/1.2430716 }\end{array}$ \\
\hline Issue Date & 2007-01 \\
\hline Doc URL & http://hdl.handle.net/2115/19108 \\
\hline Rights & Copyright $\odot 2007$ A merican Institute of Physics \\
\hline Type & article \\
\hline File Information & PF19-1.pdf \\
\hline
\end{tabular}

Instructions for use 


\title{
Integrable four-vortex motion on sphere with zero moment of vorticity
}

\author{
Takashi Sakajo ${ }^{\text {a) }}$ \\ Department of Mathematics, Hokkaido University, Kita 10 Nishi 8 Kita-ku, \\ Sapporo Hokkaido 060-8610, Japan
}

(Received 13 March 2006; accepted 7 December 2006; published online 11 January 2007)

\begin{abstract}
We consider the motion of $N$ vortex points on sphere, called the $N$-vortex problem, which is a Hamiltonian dynamical system. The three-vortex problem is integrable and its motion has already been resolved. On the other hand, when the moment of vorticity vector, which consists of weighed sums of the vortex positions, is zero at the initial moment, the four-vortex problem is integrable, but it has not been investigated yet. The present paper gives a description of the integrable four-vortex problem with the reduction method to a three-vortex problem used by Aref and Stremler. Moreover, we examine whether the vortex points collide self-similarly in finite time. The four-vortex collapse is proved to be impossible. We consider if it is possible for not all but part of the vortex points to collapse self-similarly. Moreover, we discuss the topological structure of periodic orbits obtained in the present problem. (C) 2007 American Institute of Physics. [DOI: 10.1063/1.2430716]
\end{abstract}

\section{INTRODUCTION}

We consider the two-dimensional incompressible and inviscid fluid in the surface of a sphere, for which the vorticity is a conserved quantity moving with the fluid particle. When the vorticity is discretely concentrated in a point, it is called the vortex point, which evolves like a material point. Mathematical study of the vortex-point dynamics is of importance, since it gives a theoretical basis to investigate the atmospheric flow on Earth.

Let $\left(\theta_{m}, \phi_{m}\right)$ denote the position of the $m$ th vortex point in the spherical coordinates. The motion of the $N$ vortex points with the strength $\Gamma_{m}$ on the sphere of unit radius is governed by

$$
\begin{aligned}
\dot{\theta}_{m} & =-\frac{1}{4 \pi} \sum_{j \neq m}^{N} \frac{\Gamma_{j} \sin \theta_{j} \sin \left(\phi_{m}-\phi_{j}\right)}{1-\cos \gamma_{m j}}, \\
\dot{\phi}_{m}= & -\frac{1}{4 \pi \sin \theta_{m}} \\
& \times \sum_{j \neq m}^{N} \frac{\Gamma_{j}\left[\cos \theta_{m} \sin \theta_{j} \cos \left(\phi_{m}-\phi_{j}\right)-\sin \theta_{m} \cos \theta_{j}\right]}{1-\cos \gamma_{m j}},
\end{aligned}
$$

in which $\gamma_{m j}$ represents the central angle between the $m$ th and the $j$ th vortex points, and $\cos \gamma_{m j}=\cos \Theta_{m} \cos \Theta_{j}$ $+\sin \Theta_{m} \sin \Theta_{j} \cos \left(\Psi_{m}-\Psi_{j}\right)$. With the Hamiltonian

$$
H=-\frac{1}{4 \pi} \sum_{m<j}^{N} \Gamma_{m} \Gamma_{j} \log \left(1-\cos \gamma_{m j}\right),
$$

we rewrite Eqs. (1) and (2) in the following form: ${ }^{1,2}$

$$
\frac{d \cos \theta_{m}}{d t}=\left\{\cos \theta_{m}, H\right\}, \quad \frac{d \phi_{m}}{d t}=\left\{\phi_{m}, H\right\},
$$

in which the Poisson bracket between two functions $f$ and $g$, is defined by

\footnotetext{
${ }^{a)}$ Electronic mail: sakajo@math.sci.hokudai.ac.jp
}

$$
\{f, g\}=\sum_{m=1}^{N} \frac{1}{\Gamma_{m}}\left(\frac{\partial f}{\partial \phi_{m}} \frac{\partial g}{\partial \cos \theta_{m}}-\frac{\partial g}{\partial \phi_{m}} \frac{\partial f}{\partial \cos \theta_{m}}\right) .
$$

Let us introduce the total vorticity $\Gamma=\sum_{m=1}^{N} \Gamma_{m}$ and the moment of vorticity vector $\mathbf{M}=(Q, P, S)$ by

$$
\begin{aligned}
& Q=\sum_{m=1}^{N} \Gamma_{m} \sin \theta_{m} \cos \phi_{m}, \\
& P=\sum_{m=1}^{N} \Gamma_{m} \sin \theta_{m} \sin \phi_{m}, \quad S=\sum_{m=1}^{N} \Gamma_{m} \cos \theta_{m} .
\end{aligned}
$$

They are invariant quantities, since $\{H, Q\}=\{H, P\}=\{H, S\}$ $=0$. The motion of three vortex points is integrable due to $\left\{H, P^{2}+Q^{2}\right\}=\left\{P^{2}+Q^{2}, S\right\}=0$, and it has been well investigated. ${ }^{3-5}$ Moreover, we have $\{Q, P\}=S,\{P, S\}=Q$, and $\{S, Q\}=P$, and thus they are in involution with each other when $Q=P=S=0$ holds at the initial moment. This indicates that the four-vortex problem on the sphere is integrable when the moment of vorticity is zero. This fact has been pointed out by Newton, ${ }^{2}$ but the motion has not been investigated yet.

The four-vortex motion in an unbounded plane is integrable when the total vorticity and the total impulse are zero. ${ }^{2,6}$ Aref and Stremler ${ }^{7}$ gave a complete description of the integrable system with a reduction method developed by Rott. $^{8}$ We apply their fine method to the integrable fourvortex problem on the sphere. A difference from the planar case is that zero total vorticity is unnecessary to obtain the integrability for the spherical case.

The spherical integrable four-vortex problem is of significance more than just an unsolved problem. It has recently been pointed out the relation between the periodic orbit of the $N$ vortex points and the chaotic particle mixing. ${ }^{9}$ The periodic orbit defines a two-dimensional homeomorphism on the sphere with $N$ fixed points, which are called the $N$-braids. According to Thurston-Nielsen theory, the $N$-braids are topo- 
logically classified into three types: periodic, pseudo-Anosov $(p A)$, and reducible. ${ }^{10}$ The pA map induces a complex behavior, which is known as the topological chaos. ${ }^{11}$ Since it is mathematically proved that the three-braid on sphere is always periodic, ${ }^{12,13}$ any periodic orbit obtained in the study of the integrable three-vortex problem is unable to define the pA braid. Therefore, in order to obtain the pA $N$-braids, we need to consider the $N$-vortex problem for $N \geq 4$. On the other hand, while it is generally difficult to find a periodic orbit of many vortex points, the integrable four-vortex problem provides us with infinitely many periodic orbits, which helps us find the pA braids. Furthermore, the pA four-braids are regarded as one of the simplest coherent vortex dynamics that induces the topological chaos on the sphere. Thus, the present integrable four-vortex problem is potentially applicable to a theoretical study of the chaotic particle mixing on Earth.

The article consists of six sections. In Sec. II, we reduce the four-vortex problem to a three-vortex problem following a method used by Rott in the planar problem. After discussing the existence of the self-similar collapse of the vortex points in Sec. III, we describe the integrable four-vortex motion for $\Gamma\left(\Gamma-2 \Gamma_{4}\right) \neq 0$ in Sec. IV, and for $\Gamma\left(\Gamma-2 \Gamma_{4}\right)=0$ in Sec. V. In the last section, we summarize the results and discuss the topological structure of periodic orbits.

\section{REDUCTION TO A THREE-VORTEX PROBLEM}

The method used by Rott is based on the relative configurations of the four vortex points. Let $l_{i j}^{2}=2\left(1-\cos \gamma_{i j}\right)$ represent the distance between two vortex points $\left(\theta_{i}, \phi_{i}\right)$ and $\left(\theta_{j}, \phi_{j}\right)$. The Hamiltonian $H$ and $C$ are, then, the conserved quantities.

$$
\begin{aligned}
& H=\sum_{i<j} \Gamma_{i} \Gamma_{j} \log l_{i j}^{2}, \\
& C=\Gamma^{2}-\left(P^{2}+Q^{2}+R^{2}\right)=\sum_{i<j} \Gamma_{i} \Gamma_{j} l_{i j}^{2} .
\end{aligned}
$$

Note that $C=\Gamma^{2}$, since the moment of vorticity vector is always zero.

Separating the terms for the vortex 1 and for the vortex triple 234 in $Q=P=S=0$, and squaring them, we have

$$
\begin{aligned}
& \Gamma_{1}^{2} \sin ^{2} \theta_{1} \cos ^{2} \phi_{1}=\left(\Gamma_{2} \sin \theta_{2} \cos \phi_{2}+\Gamma_{3} \sin \theta_{3} \cos \phi_{3}\right. \\
&\left.+\Gamma_{4} \sin \theta_{4} \cos \phi_{4}\right)^{2} \\
& \Gamma_{1}^{2} \sin ^{2} \theta_{1} \sin ^{2} \phi_{1}=\left(\Gamma_{2} \sin \theta_{2} \sin \phi_{2}+\Gamma_{3} \sin \theta_{3} \sin \phi_{3}\right. \\
&\left.+\Gamma_{4} \sin \theta_{4} \sin \phi_{4}\right)^{2} \\
& \Gamma_{1}^{2} \cos ^{2} \theta_{1}=\left(\Gamma_{2} \cos \theta_{2}+\Gamma_{3} \cos \theta_{3}+\Gamma_{4} \cos \theta_{4}\right)^{2}
\end{aligned}
$$

Adding both sides of (5)-(7), we define the invariant $L_{1}$ by

$$
L_{1} \equiv \Gamma_{2} \Gamma_{3} l_{23}^{2}+\Gamma_{2} \Gamma_{4} l_{24}^{2}+\Gamma_{3} \Gamma_{4} l_{34}^{2}=\left(\Gamma_{2}+\Gamma_{3}+\Gamma_{4}\right)^{2}-\Gamma_{1}^{2} .
$$

Applying the similar arithmetic to the vortex triples 134, 124, and 123, we introduce the other invariants $L_{2}, L_{3}$, and $L_{4}$ :

$$
\begin{aligned}
& L_{2} \equiv \Gamma_{3} \Gamma_{1} l_{31}^{2}+\Gamma_{1} \Gamma_{4} l_{14}^{2}+\Gamma_{3} \Gamma_{4} l_{34}^{2}=\left(\Gamma_{1}+\Gamma_{3}+\Gamma_{4}\right)^{2}-\Gamma_{2}^{2}, \\
& L_{3} \equiv \Gamma_{1} \Gamma_{2} l_{12}^{2}+\Gamma_{1} \Gamma_{4} l_{14}^{2}+\Gamma_{2} \Gamma_{4} l_{24}^{2}=\left(\Gamma_{1}+\Gamma_{2}+\Gamma_{4}\right)^{2}-\Gamma_{3}^{2}, \\
& L_{4} \equiv \Gamma_{1} \Gamma_{2} l_{12}^{2}+\Gamma_{2} \Gamma_{3} l_{23}^{2}+\Gamma_{3} \Gamma_{1} l_{31}^{2}=\left(\Gamma_{1}+\Gamma_{2}+\Gamma_{3}\right)^{2}-\Gamma_{4}^{2} .
\end{aligned}
$$

Computing $L_{1}-L_{2}-L_{3}+L_{4}, L_{1}-L_{2}+L_{3}-L_{4}$, and $L_{1}+L_{2}-L_{3}$ $-L_{4}$, we obtain the relations between distances $l_{14}^{2}, l_{24}^{2}$, and $l_{34}^{2}$, and those of the vortex triple 123 as follows:

$$
\begin{aligned}
& \Gamma_{1} \Gamma_{4} l_{14}^{2}=\Gamma_{2} \Gamma_{3} l_{23}^{2}+\Gamma\left(\Gamma_{1}-\Gamma_{2}-\Gamma_{3}+\Gamma_{4}\right), \\
& \Gamma_{2} \Gamma_{4} l_{24}^{2}=\Gamma_{3} \Gamma_{1} l_{31}^{2}+\Gamma\left(-\Gamma_{1}+\Gamma_{2}-\Gamma_{3}+\Gamma_{4}\right), \\
& \Gamma_{3} \Gamma_{4} l_{34}^{2}=\Gamma_{1} \Gamma_{2} l_{12}^{2}+\Gamma\left(-\Gamma_{1}-\Gamma_{2}+\Gamma_{3}+\Gamma_{4}\right) .
\end{aligned}
$$

Thus, the position of the fourth vortex point is derived from the configuration of the vortex triple 123 .

\section{SELF-SIMILAR COLLAPSING SOLUTION}

\section{A. Nonexistence of the four-vortex self-similar collapse}

We consider whether or not the self-similar collapse of the four vortex points is possible. The self-similar collapsing solutions have been found in the planar four-vortex problem $^{14}$ and the spherical three-vortex problem. ${ }^{4}$

Suppose that the four vortex points converge at a point in finite time; i.e., $l_{12}^{2}=l_{23}^{2}=l_{31}^{2}=l_{14}^{2}=l_{24}^{2}=l_{34}^{2}=0$. We then have $C=\Gamma^{2}=0$ at the critical time due to (4), which yields $\Gamma=0$. During the self-similar collapse, we assume that the sides of the vortex triple are related as

$$
l_{12}^{2}=\lambda_{1} l_{31}^{2}, \quad l_{23}^{2}=\lambda_{2} l_{31}^{2}, \quad \lambda_{1}, \lambda_{2} \in \mathbb{R} .
$$

It then follows from (9)-(11) with $\Gamma=0$ that we have

$$
l_{14}^{2}=\frac{\Gamma_{2} \Gamma_{3}}{\Gamma_{1} \Gamma_{4}} \lambda_{2} l_{31}^{2}, \quad l_{24}^{2}=\frac{\Gamma_{3} \Gamma_{1}}{\Gamma_{2} \Gamma_{4}} l_{31}^{2}, \quad l_{34}^{2}=\frac{\Gamma_{1} \Gamma_{2}}{\Gamma_{3} \Gamma_{4}} \lambda_{1} l_{31}^{2} .
$$

Substitution of (12) and (13) into (3) leads to

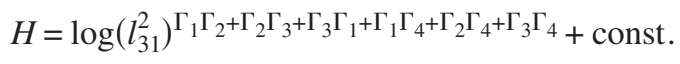

Since the Hamiltonian must remain finite as $l_{31}^{2}$ tends to zero, the necessary conditions for the self-similar collapse are given by $\Gamma=0$ and $\Gamma_{1} \Gamma_{2}+\Gamma_{2} \Gamma_{3}+\Gamma_{3} \Gamma_{1}+\Gamma_{1} \Gamma_{4}+\Gamma_{2} \Gamma_{4}+\Gamma_{3} \Gamma_{4}$ $=0$. As a matter of fact, the conditions never hold simultaneously. This is because

$$
\begin{aligned}
0 & =\Gamma_{1} \Gamma_{2}+\Gamma_{2} \Gamma_{3}+\Gamma_{3} \Gamma_{1}+\Gamma_{1} \Gamma_{4}+\Gamma_{2} \Gamma_{4}+\Gamma_{3} \Gamma_{4} \\
& =\Gamma_{1} \Gamma_{2}+\Gamma_{2} \Gamma_{3}+\Gamma_{3} \Gamma_{1}-\left(\Gamma_{1}+\Gamma_{2}+\Gamma_{3}\right)^{2} \\
& =-\frac{1}{2}\left(\Gamma_{1}+\Gamma_{2}\right)^{2}-\frac{1}{2}\left(\Gamma_{2}+\Gamma_{3}\right)^{2}-\frac{1}{2}\left(\Gamma_{3}+\Gamma_{1}\right)^{2} .
\end{aligned}
$$

This leads to $\Gamma_{1}=\Gamma_{2}=\Gamma_{3}=0$, which contradicts $\Gamma_{i} \neq 0$. Accordingly, there exists no self-similar collapse of the four vortex points. 


\section{B. Necessary conditions for the partial self-similar collapse}

There are two possibilities where not all but part of the vortex points collapse in finite time: The first case is that the three vortex points collide at a point, while the other stays at another position, which we refer to as the triple collapse. The second case is that the pairs of two vortex points collide at different positions, which we call the binary collapse. Here, we consider necessary conditions for these partial selfsimilar collapses, which will be checked in the following sections.

First suppose that the vortex triple 234 converges at a point at a certain time, i.e., $l_{23}^{2}=l_{24}^{2}=l_{34}^{2}=0$, whereas the first vortex point is located at another position; i.e., $l_{12}^{2} \neq 0, l_{31}^{2}$ $\neq 0$ and $l_{14}^{2} \neq 0$. We then have $\Gamma \neq 0$; otherwise, it follows from (9) and $l_{23}^{2}=0$ that $\Gamma_{1} \Gamma_{4} l_{14}^{2}=0$ at the collapsing time, which is a contradiction to $l_{14}^{2} \neq 0$. On the other hand, substituting (9)-(11) into (4) leads to $C=2 \Gamma_{2} \Gamma_{3} l_{23}^{2}+2 \Gamma_{2} \Gamma_{4} l_{24}^{2}$ $+2 \Gamma_{3} \Gamma_{4} l_{34}^{2}+\Gamma\left(4 \Gamma_{1}-\Gamma\right)$. Hence, we have $C=\Gamma\left(4 \Gamma_{1}-\Gamma\right)=\Gamma^{2}$ at the singular time, which yields a necessary condition $\Gamma_{2}$ $+\Gamma_{3}+\Gamma_{4}=\Gamma_{1}$ due to $\Gamma \neq 0$. It follows from the necessary condition and the self-similarity assumption, $l_{34}^{2}=\lambda_{3} l_{23}^{2}$ and $l_{24}=\lambda_{4} l_{23}^{2}$ for $\lambda_{3}, \lambda_{4} \in \mathbb{R}$, that the relations (9)-(11) become

$$
\begin{aligned}
& l_{14}^{2}=\frac{\Gamma_{2} \Gamma_{3}}{\Gamma_{1} \Gamma_{4}} l_{23}^{2}+4, \quad l_{31}^{2}=\frac{\Gamma_{2} \Gamma_{4}}{\Gamma_{3} \Gamma_{1}} \lambda_{4} l_{23}^{2}+4, \\
& l_{12}^{2}=\frac{\Gamma_{3} \Gamma_{4}}{\Gamma_{1} \Gamma_{2}} \lambda_{3} l_{23}^{2}+4 .
\end{aligned}
$$

They indicate that if the vortex triple 234 collapses, the first vortex point is located at the anti-polar position to the collapsing point. The Hamiltonian is then equivalent to

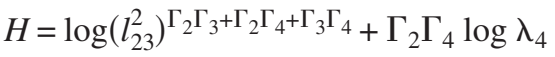

$$
\begin{aligned}
& +\Gamma_{3} \Gamma_{4} \log \lambda_{3}+\log \left(\frac{\Gamma_{2} \Gamma_{3}}{\Gamma_{1} \Gamma_{4}} l_{23}^{2}+4\right)^{\Gamma_{1} \Gamma_{4}} \\
& \times\left(\frac{\Gamma_{2} \Gamma_{4}}{\Gamma_{3} \Gamma_{1}} \lambda_{4} l_{23}^{2}+4\right)^{\Gamma_{1} \Gamma_{3}}\left(\frac{\Gamma_{3} \Gamma_{4}}{\Gamma_{1} \Gamma_{2}} \lambda_{3} l_{23}^{2}+4\right)^{\Gamma_{1} \Gamma_{2}} .
\end{aligned}
$$

Since it must remain finite as $l_{23}^{2} \rightarrow 0$, we have the necessary conditions for the self-similar collapse of the vortex triple 234 as follows:

$$
\Gamma_{2}+\Gamma_{3}+\Gamma_{4}=\Gamma_{1}, \quad \Gamma_{2} \Gamma_{3}+\Gamma_{2} \Gamma_{4}+\Gamma_{3} \Gamma_{4}=0 .
$$

In a similar manner, considering the collapse of the vortex triples 124, 134 and 123, we have the other necessary conditions for the self-similar triple collapse:

$$
\begin{array}{ll}
\Gamma_{1}+\Gamma_{2}+\Gamma_{4}=\Gamma_{3}, & \Gamma_{1} \Gamma_{2}+\Gamma_{1} \Gamma_{4}+\Gamma_{2} \Gamma_{4}=0, \\
\Gamma_{1}+\Gamma_{3}+\Gamma_{4}=\Gamma_{2}, & \Gamma_{1} \Gamma_{3}+\Gamma_{1} \Gamma_{4}+\Gamma_{3} \Gamma_{4}=0, \\
\Gamma_{1}+\Gamma_{2}+\Gamma_{3}=\Gamma_{4}, & \Gamma_{1} \Gamma_{2}+\Gamma_{2} \Gamma_{3}+\Gamma_{3} \Gamma_{1}=0 .
\end{array}
$$

Next we deal with the binary collapse. Suppose that the pairs of vortices 1 and 2, and the vortices 3 and 4 collide at different points at the same time. It then follows from $l_{12}^{2}=l_{34}^{2}=0$ at the critical time and (11) that we have either $\Gamma=0$ or $\Gamma_{1}+\Gamma_{2}=\Gamma_{3}+\Gamma_{4}$. Furthermore, it follows from the

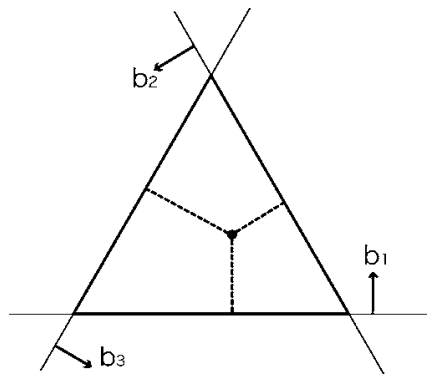

FIG. 1. Trilinear coordinates representation for the nondegenerate case.

self-similarity assumption, $l_{12}^{2}=\lambda l_{34}^{2}(\lambda \in \mathbb{R})$, that we have another necessary condition, $\Gamma_{1} \Gamma_{2}+\Gamma_{3} \Gamma_{4}=0$, since the Hamiltonian remains finite even at the singular time; that is to say, $\log \left(l_{12}^{2}\right) \Gamma_{1} \Gamma_{2}+\Gamma_{3} \Gamma_{4}<\infty$ as $l_{12}^{2} \rightarrow 0$. Thus, the necessary conditions for the binary collapse are either

$$
\Gamma=0, \quad \Gamma_{1} \Gamma_{2}+\Gamma_{3} \Gamma_{4}=0,
$$

or

$$
\Gamma_{1}+\Gamma_{2}=\Gamma_{3}+\Gamma_{4}, \quad \Gamma_{1} \Gamma_{2}+\Gamma_{3} \Gamma_{4}=0 .
$$

As a matter of fact, the condition (18) is ruled out; when $\Gamma=0$, the relations (9) and (10) are represented by

$$
\Gamma_{1} \Gamma_{4} l_{14}^{2}=\Gamma_{2} \Gamma_{3} l_{23}^{2}, \quad \Gamma_{2} \Gamma_{4} l_{24}^{2}=\Gamma_{3} \Gamma_{1} l_{31}^{2} .
$$

Since all the vortex strengths cannot have the same sign due to $\Gamma=0$, we need to consider the following two cases: (i) three strengths have the same sign and the other has the opposite sign, and (ii) the two are positive and the others are negative. For the former case, the relations in (19) are invalid. As for the latter case, $\Gamma_{1} \Gamma_{2}+\Gamma_{3} \Gamma_{4}=0$ cannot be satisfied if two of the vortices have the same sign and the other two the opposite sign. With the similar argument in terms of the other vortex pairs, we finally obtain the necessary conditions for the self-similar binary collapse:

$$
\begin{array}{ll}
\Gamma_{1}+\Gamma_{2}=\Gamma_{3}+\Gamma_{4} & \Gamma_{1} \Gamma_{2}+\Gamma_{3} \Gamma_{4}=0, \\
\Gamma_{1}+\Gamma_{3}=\Gamma_{2}+\Gamma_{4} & \Gamma_{1} \Gamma_{3}+\Gamma_{2} \Gamma_{4}=0, \\
\Gamma_{1}+\Gamma_{4}=\Gamma_{2}+\Gamma_{3} & \Gamma_{1} \Gamma_{4}+\Gamma_{2} \Gamma_{3}=0 .
\end{array}
$$

\section{FOUR-VORTEX MOTION FOR NON-DEGENERATE CASE}

\section{A. Trilinear representation and physical region}

We describe the dynamics of the four vortex points when $L_{4}=\left(\Gamma_{1}+\Gamma_{2}+\Gamma_{3}\right)^{2}-\Gamma_{4}^{2}=\Gamma\left(\Gamma-2 \Gamma_{4}\right) \neq 0$ following the work by Aref and Strelmler. ${ }^{7}$ Let us first introduce the variables

$$
b_{1}=\frac{3 \Gamma_{2} \Gamma_{3} l_{23}^{2}}{\Gamma\left(\Gamma-2 \Gamma_{4}\right)}, \quad b_{2}=\frac{3 \Gamma_{3} \Gamma_{1} l_{31}^{2}}{\Gamma\left(\Gamma-2 \Gamma_{4}\right)}, \quad b_{3}=\frac{3 \Gamma_{1} \Gamma_{2} l_{12}^{2}}{\Gamma\left(\Gamma-2 \Gamma_{4}\right)} .
$$

Since $b_{1}+b_{2}+b_{3}=3$ due to (8), the relative configuration of the vortex triple 123 corresponds to a point in the phase plane as in Fig. 1. Each component of $\left(b_{1}, b_{2}, b_{3}\right)$ represents the distance from one of the sides of the equilateral triangle 
with height 3 , which is called the trilinear triangle. The trilinear representation is a standard tool to give a description of the integrable three-vortex motion. ${ }^{3,5,7,15}$

In the four-vortex problem, we need to consider the additional distances between the vortex triple 123 and the fourth vortex point. We define the other variables $B_{1}, B_{2}$, and $B_{3}$ from (9)-(11) as follows:

$$
\begin{aligned}
& B_{1}=\frac{3 \Gamma_{1} \Gamma_{4} l_{14}^{2}}{\Gamma\left(\Gamma-2 \Gamma_{4}\right)}=b_{1}+\frac{3}{\Gamma-2 \Gamma_{4}}\left(\Gamma-2 \Gamma_{2}-2 \Gamma_{3}\right), \\
& B_{2}=\frac{3 \Gamma_{2} \Gamma_{4} l_{24}^{2}}{\Gamma\left(\Gamma-2 \Gamma_{4}\right)}=b_{2}+\frac{3}{\Gamma-2 \Gamma_{4}}\left(\Gamma-2 \Gamma_{3}-2 \Gamma_{1}\right), \\
& B_{3}=\frac{3 \Gamma_{3} \Gamma_{4} l_{34}^{2}}{\Gamma\left(\Gamma-2 \Gamma_{4}\right)}=b_{3}+\frac{3}{\Gamma-2 \Gamma_{4}}\left(\Gamma-2 \Gamma_{1}-2 \Gamma_{2}\right) .
\end{aligned}
$$

Since $B_{1}+B_{2}+B_{3}=6 \Gamma_{4} /\left(\Gamma-2 \Gamma_{4}\right)$, the triple $\left(B_{1}, B_{2}, B_{3}\right)$ also defines another trilinear coordinates; Each component $B_{i}$ represents the distance from one of the sides of the equilateral triangle with height $6 \Gamma_{4} /\left(\Gamma-2 \Gamma_{4}\right)$, which is called the physical triangle.

Since the vortex triple 123 forms a triangle on the sphere, the relative distances are restricted by the triangle inequality, ${ }^{3}$

$$
2 l_{12}^{2} l_{23}^{2}+2 l_{23}^{2} l_{31}^{2}+2 l_{31}^{2} l_{12}^{2}-l_{12}^{4}-l_{23}^{4}-l_{31}^{4} \geq l_{12}^{2} l_{23}^{2} l_{31}^{2} .
$$

In terms of $b_{i}$, it is represented by

$$
3 V_{p}-\Gamma\left(\Gamma-2 \Gamma_{4}\right) b_{1} b_{2} b_{3} \geq 0,
$$

where $\quad V_{p}=2 \Gamma_{2} \Gamma_{3} b_{2} b_{3}+2 \Gamma_{3} \Gamma_{1} b_{3} b_{1}+2 \Gamma_{1} \Gamma_{2} b_{1} b_{2}-\left(\Gamma_{1} b_{1}\right)^{2}$ $-\left(\Gamma_{2} b_{2}\right)^{2}-\left(\Gamma_{3} b_{3}\right)^{2}$. The region where the condition (27) is satisfied is called the physical region. Any point at the boundary of the physical region corresponds to the configuration where the three vortex points lie on a great circle of the sphere. We then note that the fourth vortex point is also on the same great circle due to $P=Q=S=0$.

Now, we consider the singular points at the boundary of the physical region, for which two vortex points coincide. They are obtained by solving

$$
b_{1}+b_{2}+b_{3}=3, \quad 3 V_{p}-\Gamma\left(\Gamma-2 \Gamma_{4}\right) b_{1} b_{2} b_{3}=0,
$$

and $b_{i}=0$. Thus, we have the three singular points in the trilinear coordinates:

$$
\begin{aligned}
& \left(b_{1}, b_{2}, b_{3}\right)=\left(0, \frac{3 \Gamma_{3}}{\Gamma_{2}+\Gamma_{3}}, \frac{3 \Gamma_{2}}{\Gamma_{2}+\Gamma_{3}}\right), \\
& \left(b_{1}, b_{2}, b_{3}\right)=\left(\frac{3 \Gamma_{3}}{\Gamma_{1}+\Gamma_{3}}, 0, \frac{3 \Gamma_{1}}{\Gamma_{1}+\Gamma_{3}}\right), \\
& \left(b_{1}, b_{2}, b_{3}\right)=\left(\frac{3 \Gamma_{2}}{\Gamma_{1}+\Gamma_{2}}, \frac{3 \Gamma_{1}}{\Gamma_{1}+\Gamma_{2}}, 0\right) .
\end{aligned}
$$

Since the boundary of the physical region is a conic section, the points are tangent to the sides of the trilinear triangle. Thus, we refer to the points as the points of tangency.

Finally, we obtain the points tangent to the sides of the physical triangle. Solving Eqs. (28) with $B_{i}=0$, we have

$$
\begin{aligned}
& \left(b_{1}, b_{2}, b_{3}\right)=\left(\frac{3\left(2 \Gamma_{2}+2 \Gamma_{3}-\Gamma\right)}{\Gamma-2 \Gamma_{4}}, \frac{3 \Gamma_{1}\left(2 \Gamma_{2}-\Gamma\right)}{\left(\Gamma-2 \Gamma_{4}\right)\left(\Gamma_{2}+\Gamma_{3}-\Gamma\right)},\right. \\
& \left.\frac{3 \Gamma_{1}\left(2 \Gamma_{3}-\Gamma\right)}{\left(\Gamma-2 \Gamma_{4}\right)\left(\Gamma_{2}+\Gamma_{3}-\Gamma\right)}\right) \text {. } \\
& \left(b_{1}, b_{2}, b_{3}\right)=\left(\frac{3 \Gamma_{2}\left(2 \Gamma_{1}-\Gamma\right)}{\left(\Gamma-2 \Gamma_{4}\right)\left(\Gamma_{3}+\Gamma_{1}-\Gamma\right)}, \frac{3\left(2 \Gamma_{3}+2 \Gamma_{1}-\Gamma\right)}{\Gamma-2 \Gamma_{4}},\right. \\
& \left.\frac{3 \Gamma_{2}\left(2 \Gamma_{3}-\Gamma\right)}{\left(\Gamma-2 \Gamma_{4}\right)\left(\Gamma_{3}+\Gamma_{1}-\Gamma\right)}\right), \\
& \left(b_{1}, b_{2}, b_{3}\right)=\left(\frac{3 \Gamma_{3}\left(2 \Gamma_{1}-\Gamma\right)}{\left(\Gamma-2 \Gamma_{4}\right)\left(\Gamma_{1}+\Gamma_{2}-\Gamma\right)},\right. \\
& \left.\frac{3 \Gamma_{3}\left(2 \Gamma_{2}-\Gamma\right)}{\left(\Gamma-2 \Gamma_{4}\right)\left(\Gamma_{1}+\Gamma_{2}-\Gamma\right)}, \frac{3\left(2 \Gamma_{1}+2 \Gamma_{2}-\Gamma\right)}{\Gamma-2 \Gamma_{4}}\right) \text {. }
\end{aligned}
$$

These points (32)-(34) represent the singular configurations where the vortices 1 and 4,2 and 4, and 3 and 4 coincide, respectively.

As the four vortex points evolve, the corresponding point in the trilinear phase space moves along a contour curve of the Hamiltonian,

$$
\begin{aligned}
H= & \Gamma_{2} \Gamma_{3} \log \left|b_{1}\right|+\Gamma_{3} \Gamma_{1} \log \left|b_{2}\right|+\Gamma_{1} \Gamma_{2} \log \left|b_{3}\right| \\
& +\Gamma_{1} \Gamma_{4} \log \left|B_{1}\right|+\Gamma_{2} \Gamma_{4} \log \left|B_{2}\right|+\Gamma_{3} \Gamma_{4} \log \left|B_{3}\right| .
\end{aligned}
$$

Therefore, in order to observe the four-vortex motion, we plot the contour curves of the Hamiltonian inside the physical region with the points of tangency at the boundary.

\section{B. Restriction on the vortex strengths}

Unlike the planar four-vortex problem, ${ }^{7}$ the zero total vorticity condition is unnecessary to guarantee the integrability in the present spherical problem. Nevertheless, as a matter of fact, the vortex strengths are restricted, since the distances between two vortex points are bounded by $0 \leq l_{i j}^{2} \leq 4$, whereas they are unbounded for the planar case. Thus, in what follows, we consider the existence region of $\Gamma_{3}$ and $\Gamma_{4}$ for the specified $\Gamma_{1}$ and $\Gamma_{2}$, which is referred to as the possible region. Without loss of generality, we may assume that $\Gamma_{1} \geq \Gamma_{2} \geq \Gamma_{3} \geq \Gamma_{4}$. Besides, noting that Eqs. (1) and (2) are symmetric with respect to the discrete transformation $\Gamma_{i} \rightarrow-\Gamma_{i}$ and $t \rightarrow-t$, it is sufficient to deal with the following three cases:

$$
\begin{aligned}
& \Gamma_{1} \geq \Gamma_{2} \geq \Gamma_{3} \geq \Gamma_{4}>0, \quad \Gamma_{1} \geq \Gamma_{2} \geq \Gamma_{3}>0>\Gamma_{4}, \\
& \Gamma_{1} \geq \Gamma_{2}>0>\Gamma_{3} \geq \Gamma_{4} .
\end{aligned}
$$

In the meantime, we consider the singular cases when the points of tangency (29)-(34) take the same position. They play an important role in the description of the fourvortex motion, since the change of the distribution of the points results in the change of the topological structure of the contour lines of the Hamiltonian. Substituting $b_{i}=0$ into (32)-(34), we obtain the following six singular cases: 
(a)

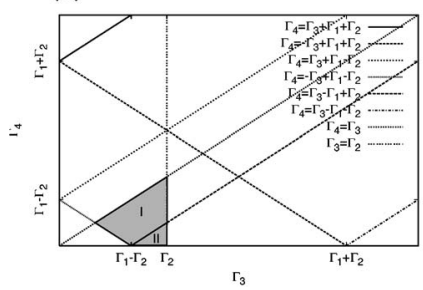

(b)

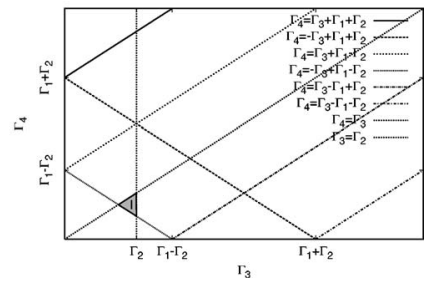

FIG. 2. The possible regions for $\Gamma_{1} \geq \Gamma_{2} \geq \Gamma_{3} \geq \Gamma_{4}>0$ with (a) $\Gamma_{1}-\Gamma_{2}$ $\leq \Gamma_{2}$ and (b) $\Gamma_{2}<\Gamma_{1}-\Gamma_{2} \leq 2 \Gamma_{2}$.

$\Gamma_{1}=\Gamma_{2}+\Gamma_{3}+\Gamma_{4}, \quad \Gamma_{3}=\Gamma_{1}+\Gamma_{2}+\Gamma_{4}, \quad \Gamma_{2}=\Gamma_{1}+\Gamma_{3}+\Gamma_{4}$,

$\Gamma_{1}+\Gamma_{2}=\Gamma_{3}+\Gamma_{4}, \quad \Gamma_{1}+\Gamma_{3}=\Gamma_{2}+\Gamma_{4}, \quad \Gamma_{1}+\Gamma_{4}=\Gamma_{2}+\Gamma_{3}$.

Note that they are the part of the necessary conditions for the triple collapse (14)-(16) and for the binary collapse (20)-(22).

\section{Case I: $\Gamma_{1} \geq \Gamma_{2} \geq \Gamma_{3} \geq \Gamma_{4}>0$}

We derive the conditions of the vortex strengths so that the invariants $L_{i}$ are well defined. Since $\min L_{i}=0$ due to $\Gamma_{i}>0$, the necessary conditions are given by

$$
\begin{aligned}
& L_{1}=\left(\Gamma_{2}+\Gamma_{3}+\Gamma_{4}\right)^{2}-\Gamma_{1}^{2}>0, \\
& L_{2}=\left(\Gamma_{1}+\Gamma_{3}+\Gamma_{4}\right)^{2}-\Gamma_{2}^{2}>0, \\
& L_{3}=\left(\Gamma_{1}+\Gamma_{2}+\Gamma_{4}\right)^{2}-\Gamma_{3}^{2}>0, \\
& L_{4}=\left(\Gamma_{1}+\Gamma_{2}+\Gamma_{3}\right)^{2}-\Gamma_{4}^{2}>0 .
\end{aligned}
$$

Because $\Gamma_{1} \geq \Gamma_{2} \geq \Gamma_{3} \geq \Gamma_{4}>0$, they are reduced to

$$
\Gamma_{1}<\Gamma_{2}+\Gamma_{3}+\Gamma_{4}, \quad 0<\Gamma_{4} \leq \Gamma_{3} \leq \Gamma_{2} .
$$

Strictly speaking, it is necessary to show that (37) assures $L_{i}<\max L_{i}$. The proof of the upper bound is important but lengthy, so we give it in the appendix. Figure 2 shows the possible regions for $\Gamma_{1}-\Gamma_{2} \leq \Gamma_{2}$ and for $\Gamma_{2}<\Gamma_{1}-\Gamma_{2} \leq 2 \Gamma_{2}$.
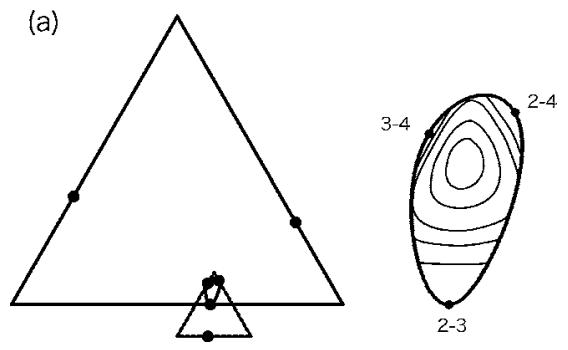

(b)

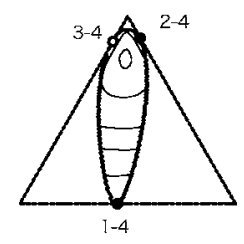

FIG. 3. The physical region and the contour lines of the Hamiltonian for (a) $\Gamma_{1}=5, \Gamma_{2}=3, \Gamma_{3}=2$, and $\Gamma_{4}=1$ and (b) $\Gamma_{1}=5, \Gamma_{2}=3, \Gamma_{3}=\frac{5}{2}$, and $\Gamma_{1}=\frac{1}{4}$.

No possible region exists for $\Gamma_{1}-\Gamma_{2}>2 \Gamma_{2}$. The singular line $\Gamma_{1}+\Gamma_{4}=\Gamma_{2}+\Gamma_{3}$ cuts the possible region into two regions I and II for $\Gamma_{1}-\Gamma_{2} \leq \Gamma_{2}$. The possible region for $\Gamma_{1}-\Gamma_{2}>\Gamma_{2}$ is still denoted by the region $\mathrm{I}$, since the distribution of the points of tangency is the same as that for $\Gamma_{1}-\Gamma_{2} \leq \Gamma_{2}$. We give examples of the contour lines of the Hamiltonian, when we choose the vortex strengths $\Gamma_{3}$ and $\Gamma_{4}$ from regions I and II for $\Gamma_{1}=5$ and $\Gamma_{2}=3$.

Figure 3(a) shows the contour lines for $\Gamma_{1}=5, \Gamma_{2}=3$, $\Gamma_{3}=2$, and $\Gamma_{4}=1$ in region $\mathrm{I}$. The trilinear triangle and the physical triangle are plotted by a solid line and a dotted line, respectively. Since the physical region is too small to discern, we magnify it to the right. The points of tangency at the boundary of the physical region are designated by $2-3,2-4$, and 3-4, which correspond to (29), (33), and (34), respectively. The topological structure of the contour curves around the singular points is elliptic. There is an elliptic center inside the physical region, which corresponds to a relative fixed configuration.

Figure 3(b) shows the contour lines for $\Gamma_{1}=5, \Gamma_{2}=3$, $\Gamma_{3}=\frac{5}{2}$, and $\Gamma_{4}=\frac{1}{4}$ in region II. We just show the magnified physical region in the physical triangle, since the trilinear triangle is too large to show in the figure. When we compare the figure with Fig. 3(a), the point of tangency $2-3$ at the boundary is replaced by $1-4$, but the topological structure of the contour curves is similar.

When the vortex strengths $\Gamma_{3}$ and $\Gamma_{4}$ are on the singular boundary, i.e., $\Gamma_{1}+\Gamma_{4}=\Gamma_{2}+\Gamma_{3}$, the binary collapse is possible, since it is one of the necessary conditions in (22). (a)

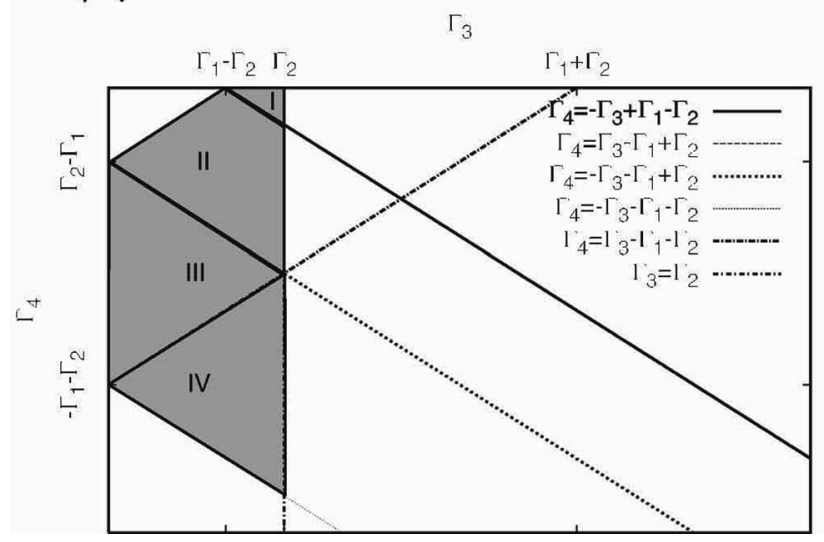

(b)

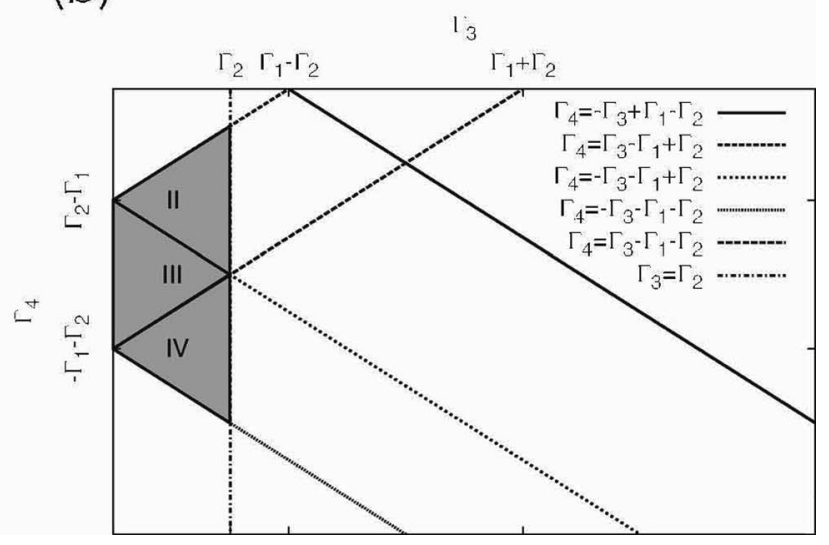

FIG. 4. The possible regions for $\Gamma_{1} \geq \Gamma_{2} \geq \Gamma_{3}>0>\Gamma_{4}$ with (a) $\Gamma_{1}-\Gamma_{2}<\Gamma_{2}$ and (b) $\Gamma_{2}<\Gamma_{1}-\Gamma_{2}$. 
(a)

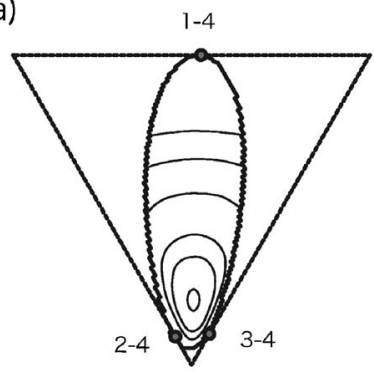

(b)

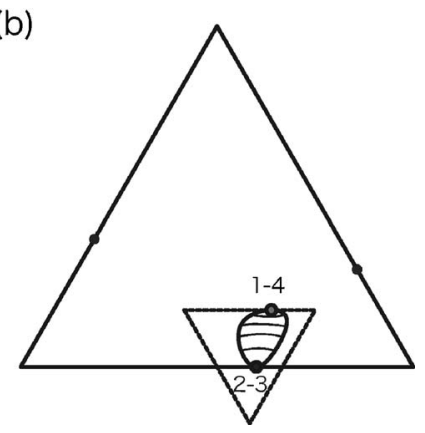

FIG. 5. The contour lines of the Hamiltonian for (a) $\Gamma_{1}=5, \Gamma_{2}=3, \Gamma_{3}=\frac{9}{4}$, and $\Gamma_{4}=-\frac{1}{4}$ and (b) $\Gamma_{1}=5, \Gamma_{2}=3, \Gamma_{3}=2$, and $\Gamma_{4}=-2$.

However, the other necessary condition, i.e., $\Gamma_{1} \Gamma_{4}+\Gamma_{2} \Gamma_{3}=0$, never holds, since all the vortex strengths are positive. Hence, no binary collapse is possible in this case.

\section{Case II: $\Gamma_{1} \geq \Gamma_{2} \geq \Gamma_{3}>0>\Gamma_{4}$}

Since $\Gamma_{i} \Gamma_{4}$ for $i=1,2,3$ are negative due to $\Gamma_{4}<0$, we have $\min L_{1}=4 \Gamma_{3} \Gamma_{4}+4 \Gamma_{2} \Gamma_{4}, \quad \min L_{2}=4 \Gamma_{1} \Gamma_{4}+4 \Gamma_{3} \Gamma_{4}$, $\min L_{3}=4 \Gamma_{1} \Gamma_{4}+4 \Gamma_{2} \Gamma_{4}$, and $\min L_{4}=0$. Hence, the vortex strengths should satisfy

$$
\begin{aligned}
& 4 \Gamma_{3} \Gamma_{4}+4 \Gamma_{2} \Gamma_{4}<\left(\Gamma_{2}+\Gamma_{3}+\Gamma_{4}\right)^{2}-\Gamma_{1}^{2}, \\
& 4 \Gamma_{1} \Gamma_{4}+4 \Gamma_{3} \Gamma_{4}<\left(\Gamma_{1}+\Gamma_{3}+\Gamma_{4}\right)^{2}-\Gamma_{2}^{2}, \\
& 4 \Gamma_{1} \Gamma_{4}+4 \Gamma_{2} \Gamma_{4}<\left(\Gamma_{1}+\Gamma_{2}+\Gamma_{4}\right)^{2}-\Gamma_{3}^{2}, \\
& 0<\left(\Gamma_{1}+\Gamma_{2}+\Gamma_{3}\right)^{2}-\Gamma_{4}^{2} .
\end{aligned}
$$

Because $\Gamma_{1} \geq \Gamma_{2} \geq \Gamma_{3}>0>\Gamma_{4}$, they are reduced to

$$
\begin{aligned}
& \Gamma_{1}<\Gamma_{2}+\Gamma_{3}-\Gamma_{4}, \quad-\Gamma_{4}<\Gamma_{1}+\Gamma_{2}+\Gamma_{3}, \\
& \Gamma_{4}<0<\Gamma_{3} \leq \Gamma_{2} .
\end{aligned}
$$

The possible region is shown in Fig. 4, which is divided into four regions I to IV by the singular lines (35) for $\Gamma_{1}-\Gamma_{2}$ $\leq \Gamma_{2}$, and three regions for $\Gamma_{1}-\Gamma_{2}>\Gamma_{2}$.

Figure 5(a) shows the contour lines for $\Gamma_{1}=5, \Gamma_{2}=3$, $\Gamma_{3}=\frac{9}{4}$, and $\Gamma_{4}=-\frac{1}{4}$ in region I. It is the same as Fig. 3(a), when it is turned upside down. Figure 5(b) shows the contour lines for $\Gamma_{1}=5, \Gamma_{2}=3, \Gamma_{3}=2$, and $\Gamma_{4}=-2$ in the region II. As the vortex strengths $\Gamma_{3}$ and $\Gamma_{4}$ pass the singular boundary $\Gamma_{2}+\Gamma_{3}+\Gamma_{4}=\Gamma_{1}$ between regions I and II, the points of tangency 2-4 and 3-4 are united, and the new point of tangency $2-3$ emerges. The topological structure of the contour is simple.

When $\Gamma_{1}=5, \Gamma_{2}=3, \Gamma_{3}=2$, and $\Gamma_{4}=-5$ in region III, the contour lines are given in Fig. 6(a). On crossing the singular boundary $\Gamma_{1}+\Gamma_{3}+\Gamma_{4}=\Gamma_{2}$ between regions II and III, the point of tangency 1-4 observed in region II splits into two elliptic points of tangency $1-3$ and 3-4. There is a hyperbolic fixed point inside the physical region, which is connected by homoclinic orbits. The topological structure suggests that there exists another elliptic fixed point at the boundary near the side $B_{1}=0$. The contour lines for $\Gamma_{1}=5$, $\Gamma_{2}=3, \Gamma_{3}=2$, and $\Gamma_{4}=-8$ in region IV are shown in Fig. 6(b).

(a)

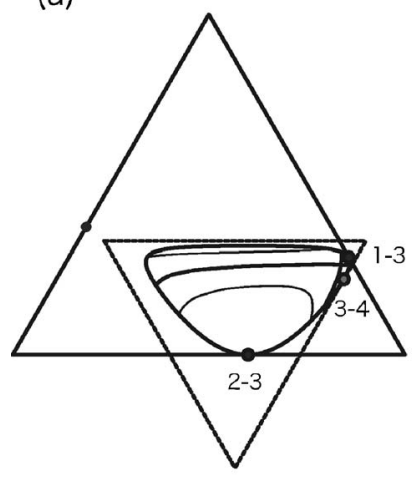

(b)

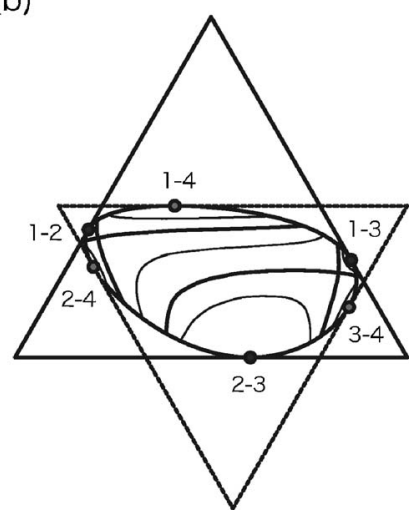

FIG. 6. The physical region and the contour lines for (a) $\Gamma_{1}=5, \Gamma_{2}=3, \Gamma_{3}$ $=2$, and $\Gamma_{4}=-5$ and (b) $\Gamma_{1}=5, \Gamma_{2}=3, \Gamma_{3}=2$, and $\Gamma_{4}=-8$.

The boundary of the physical region touches the side $b_{3}=0$, and the three points of tangency $1-2,2-4$, and 1-4 emerge. There are two hyperbolic fixed points with homoclinic connections inside the physical region.

We examine whether the self-similar triple collapse is possible or not when we choose the vortex strengths at the singular boundaries (35) in the possible regions. The corresponding necessary conditions are given in (14)-(16). We examine each of them separately. First, we show that there exists no triple collapse for (15) and (16); It follows from (15) that we obtain $\Gamma_{3}=\left(\Gamma_{1}^{2}+\Gamma_{1} \Gamma_{2}+\Gamma_{2}^{2}\right) /\left(\Gamma_{1}+\Gamma_{2}\right)$. Then, since $\Gamma_{2}-\Gamma_{3}=-\Gamma_{1}^{2} /\left(\Gamma_{1}+\Gamma_{2}\right)<0$, it contradicts the assumption $\Gamma_{2} \geq \Gamma_{3}$. As for the necessary conditions in (16), they give the equation of $\Gamma_{3}$; i.e., $\Gamma_{3}^{2}+\Gamma_{3}\left(\Gamma_{1}-\Gamma_{2}\right)+\Gamma_{1}\left(\Gamma_{1}-\Gamma_{2}\right)$ $=0$. Since the determinant of the quadratic equation is $\left(3 \Gamma_{1}\right.$ $\left.+\Gamma_{2}\right)\left(\Gamma_{2}-\Gamma_{1}\right)<0$, there is no real solution of the equation and thus no self-similar triple collapse. Next, regarding the conditions in (14), we are unable to rule out the possibility of the triple collapse. As a matter of fact, $\Gamma_{1}=6, \Gamma_{2}=5$, $\Gamma_{3}=\frac{1}{2}(1+\sqrt{21})$ and $\Gamma_{4}=\frac{1}{2}(1-\sqrt{21})$ satisfy the necessary conditions. Hence, in order to conclude that the triple collapse really occurs, we have to study this case more carefully.

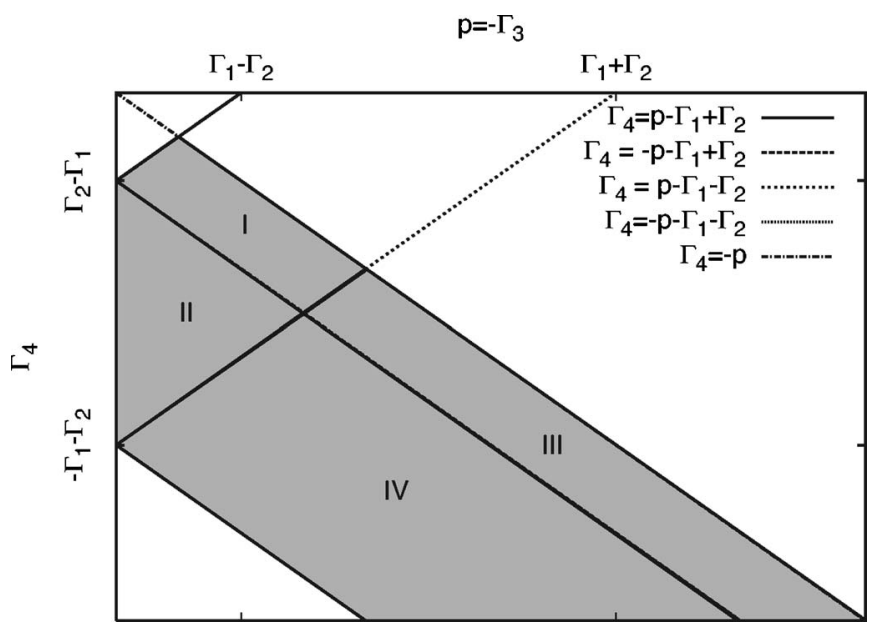

FIG. 7. The possible region for $\Gamma_{1} \geq \Gamma_{2}>0>\Gamma_{3} \geq \Gamma_{4}$. 
(a)

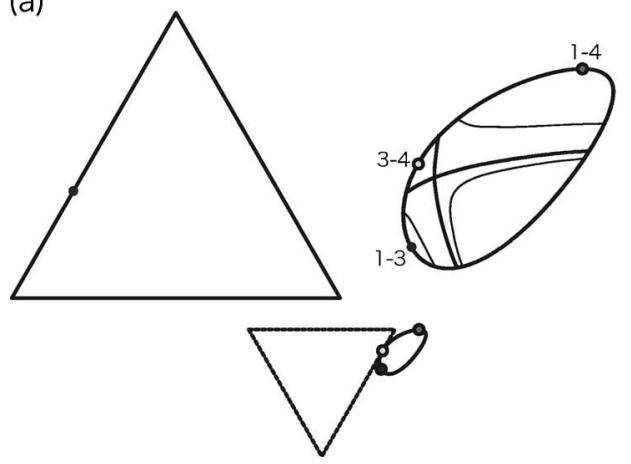

(b)

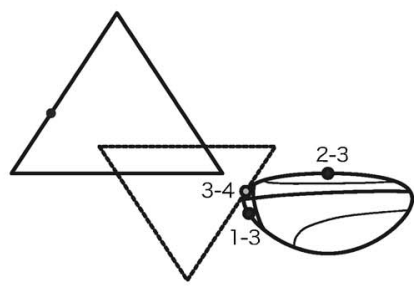

FIG. 8. The contour lines of the Hamiltonian for (a) $\Gamma_{1}=5, \quad \Gamma_{2}=3$, $\Gamma_{3}=-1$, and $\Gamma_{4}=-2$ and (b) $\Gamma_{1}=5$, $\Gamma_{2}=3, \Gamma_{3}=-1$, and $\Gamma_{4}=-5$.

\section{E. Case III: $\Gamma_{1} \geq \Gamma_{2}>0>\Gamma_{3} \geq \Gamma_{4}$}

Since $\min L_{1}=4 \Gamma_{2} \Gamma_{3}+4 \Gamma_{2} \Gamma_{4}, \quad \min L_{2}=4 \Gamma_{1} \Gamma_{3}+4 \Gamma_{1} \Gamma_{4}$, $\min L_{3}=4 \Gamma_{1} \Gamma_{4}+4 \Gamma_{2} \Gamma_{4}$, and $\min L_{4}=4 \Gamma_{1} \Gamma_{3}+4 \Gamma_{2} \Gamma_{3}$, the vortex strengths should satisfy the following conditions:

$$
\begin{aligned}
& \min L_{1}<\left(\Gamma_{2}+\Gamma_{3}+\Gamma_{4}\right)^{2}-\Gamma_{1}^{2}, \\
& \min L_{2}<\left(\Gamma_{1}+\Gamma_{3}+\Gamma_{4}\right)^{2}-\Gamma_{2}^{2}, \\
& \min L_{3}<\left(\Gamma_{1}+\Gamma_{2}+\Gamma_{4}\right)^{2}-\Gamma_{3}^{2}, \\
& \min L_{4}<\left(\Gamma_{1}+\Gamma_{2}+\Gamma_{3}\right)^{2}-\Gamma_{4}^{2} .
\end{aligned}
$$

Hence, the possible region is described by

$$
\begin{aligned}
& \Gamma_{3}+\Gamma_{4}-\Gamma_{2}+\Gamma_{1}<0, \quad \Gamma_{1}+\Gamma_{2}-\Gamma_{3}+\Gamma_{4}>0, \\
& \Gamma_{4} \leq \Gamma_{3}<0 .
\end{aligned}
$$

Defining $p=-\Gamma_{3}>0$ for convenience, we draw the possible region of $\left(p, \Gamma_{4}\right)$ in Fig. 7 . It consists of the four subregions I, II, III, and IV, separated by the singular boundaries $\Gamma_{1}+\Gamma_{4}=\Gamma_{2}+\Gamma_{3}$ and $\Gamma_{4}=-\Gamma_{3}-\Gamma_{1}-\Gamma_{2}$.

When $\Gamma_{1}=5, \Gamma_{2}=3, \Gamma_{3}=-1$, and $\Gamma_{4}=-2$ in region $\mathrm{I}$, the physical region is located outside of the trilinear triangle and the physical triangle as in Fig. 8(a). There are three elliptic points of tangency $1-3,1-4$, and $3-4$, an elliptic fixed point at the boundary and a hyperbolic fixed point with homoclinic connections inside the physical region. Figure 8(b) shows the contour lines for $\Gamma_{1}=5, \Gamma_{2}=3, \Gamma_{3}=-1$, and $\Gamma_{4}=-5$ in region II. The topological structure is the same as that in the region I except for the point of tangency 2-3.

Figures 9(a) and 9(b) show the contour lines for $\Gamma_{1}=5$, $\Gamma_{2}=3, \Gamma_{3}=-2$, and $\Gamma_{4}=-8$ in region $\mathrm{IV}$, and for $\Gamma_{1}=5$,
$\Gamma_{2}=3, \Gamma_{3}=-4$, and $\Gamma_{4}=-5$ in region III. The topological structures of the contour curves are quite similar except for the points of tangency at the boundary of the physical region.

The singular boundaries in the possible region are represented by $\Gamma_{4}=p-\Gamma_{1}-\Gamma_{2}$ and $\Gamma_{4}=-p-\Gamma_{1}+\Gamma_{2}$. It is unnecessary to deal with the first case due to $\Gamma \neq 0$. As for $\Gamma_{4}=-p$ $-\Gamma_{1}+\Gamma_{2}$, the other necessary condition in (22), i.e., $\Gamma_{1} \Gamma_{4}$ $+\Gamma_{2} \Gamma_{3}=0$, never holds, since it is negative due to $\Gamma_{1} \geq \Gamma_{2}$ $>0>\Gamma_{3} \geq \Gamma_{4}$. Consequently, the self-similar binary collapse is impossible.

\section{FOUR-VORTEX MOTION FOR DEGENERATE CASE}

\section{A. Trilinear coordinates and the physical region}

For the degenerate case $L_{4}=0$, the trilinear coordinates are defined by $b_{1}=\Gamma_{2} \Gamma_{3} l_{23}^{2}, b_{2}=\Gamma_{3} \Gamma_{1} l_{31}^{2}$, and $b_{3}=\Gamma_{1} \Gamma_{2} l_{12}^{2}$, which satisfy $b_{1}+b_{2}+b_{3}=0$. Then, the trilinear coordinates $\left(b_{1}, b_{2}, b_{3}\right)$ define a point in the planar phase space, as shown in Fig. 10. The physical region is derived from

$$
\begin{aligned}
& 2 \Gamma_{1} \Gamma_{2} b_{1} b_{2}+2 \Gamma_{2} \Gamma_{3} b_{2} b_{3}+2 \Gamma_{3} \Gamma_{1} b_{3} b_{1}-\left(\Gamma_{1} b_{1}\right)^{2}-\left(\Gamma_{2} b_{2}\right)^{2} \\
& \quad-\left(\Gamma_{3} b_{3}\right)^{2} \geq b_{1} b_{2} b_{3} .
\end{aligned}
$$

The variables $B_{1}, B_{2}$, and $B_{3}$ are also defined by

$$
\begin{aligned}
& B_{1} \equiv \Gamma_{1} \Gamma_{4} l_{14}^{2}=b_{1}+\Gamma\left(\Gamma_{1}-\Gamma_{2}-\Gamma_{3}+\Gamma_{4}\right), \\
& B_{2} \equiv \Gamma_{2} \Gamma_{4} l_{24}^{2}=b_{2}+\Gamma\left(-\Gamma_{1}+\Gamma_{2}-\Gamma_{3}+\Gamma_{4}\right), \\
& B_{3} \equiv \Gamma_{3} \Gamma_{4} l_{34}^{2}=b_{3}+\Gamma\left(-\Gamma_{1}-\Gamma_{2}+\Gamma_{3}+\Gamma_{4}\right) .
\end{aligned}
$$

We show the contour lines of the Hamiltonian in the physical region for $\Gamma=0$ and $\Gamma=2 \Gamma_{4}$ separately in the following subsections. (a)

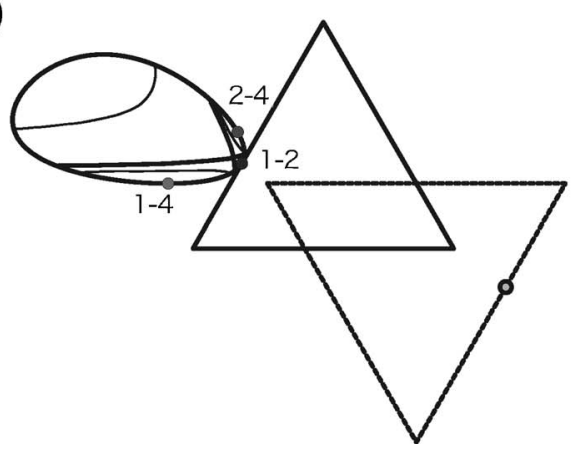

(b)

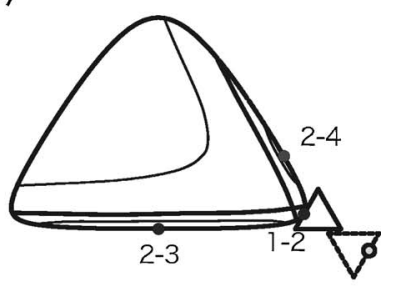

FIG. 9. The contour lines of the Hamiltonian for (a) $\Gamma_{1}=5, \quad \Gamma_{2}=3$, $\Gamma_{3}=-2$, and $\Gamma_{4}=-8$ and (b) $\Gamma_{1}=5$, $\Gamma_{2}=3, \Gamma_{3}=-4$, and $\Gamma_{4}=-5$. 


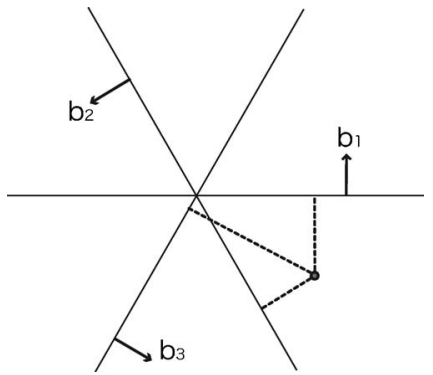

FIG. 10. Trilinear coordinates representation for the degenerate case.

\section{B. Case I: $\Gamma=0$}

For $\Gamma=0$, we have $b_{i}=B_{i}$ due to (39)-(41). The point of tangency at the boundary is the origin of the degenerate trilinear phase space. While the relative distances $l_{12}^{2}, l_{23}^{2}$, and $l_{31}^{2}$ have been already restricted by the triangle inequality (38), the other distances $l_{14}^{2}, l_{24}^{2}$, and $l_{34}^{2}$ defined by (39)-(41) should be bounded. Thus, we have the following conditions of the vortex strengths:

$$
0<\frac{\Gamma_{2} \Gamma_{3}}{\Gamma_{1} \Gamma_{4}} l_{23}^{2}, \quad \frac{\Gamma_{3} \Gamma_{1}}{\Gamma_{2} \Gamma_{4}} l_{31}^{2}, \quad \frac{\Gamma_{1} \Gamma_{2}}{\Gamma_{3} \Gamma_{4}} l_{12}^{2} \leq 4 .
$$

Since the strength $\Gamma_{4}$ is given by $\Gamma_{4}=-\Gamma_{1}-\Gamma_{2}-\Gamma_{3}$, we consider the condition of the three vortex strengths for the following three cases: (i) $\Gamma_{1} \geq \Gamma_{2} \geq \Gamma_{3}>0$, (ii) $\Gamma_{1} \geq \Gamma_{2}>0$ $>\Gamma_{3}$, and (iii) $\Gamma_{1}>0>\Gamma_{2} \geq \Gamma_{3}$. For the first case, since $\left(\Gamma_{2} \Gamma_{3} / \Gamma_{1} \Gamma_{4}\right) l_{23}^{2}<0$ due to $\Gamma_{4}<0$ and $l_{23}^{2} \geq 0$, the condition (42) is not satisfied. For the second case, $\Gamma_{4}$ must be negative; otherwise, $\left(\Gamma_{2} \Gamma_{3} / \Gamma_{1} \Gamma_{4}\right) l_{23}^{2}<0$. With similar reasoning, $\Gamma_{4}$ is positive for the third case. Remembering that Eqs. (1) and (2) have symmetry with respect to the sign changing of the vortex strengths and the time reversal, we have only to treat just one case, $\Gamma_{1} \geq \Gamma_{2}>0>\Gamma_{3}$ and $\Gamma_{4}<0$; i.e., $\Gamma_{3}>-\Gamma_{1}-\Gamma_{2}$.

Figure 11 shows the contour lines of the Hamiltonian for $\Gamma_{1}=4, \Gamma_{2}=2, \Gamma_{3}=-3$, and $\Gamma_{4}=-3$. The origin corresponds to the four-vortex collision. The topological structure of the contour is simple; There is an elliptic fixed point at the boundary.

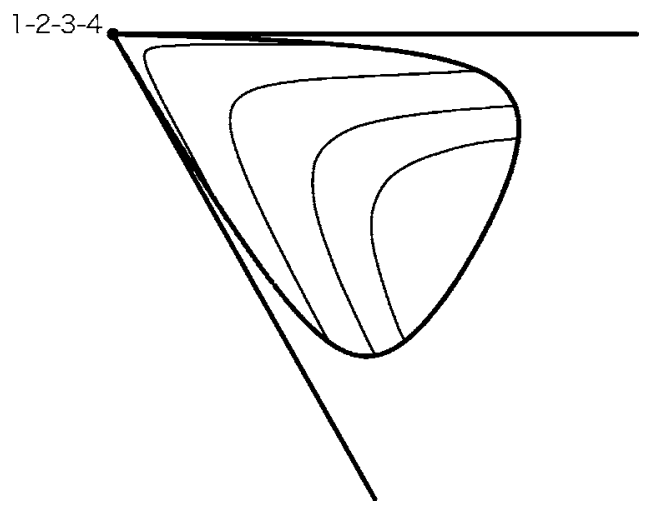

FIG. 11. The physical region and the contour lines of the Hamiltonian for $\Gamma_{1}=4, \Gamma_{2}=2, \Gamma_{3}=-3$, and $\Gamma_{4}=-3$.
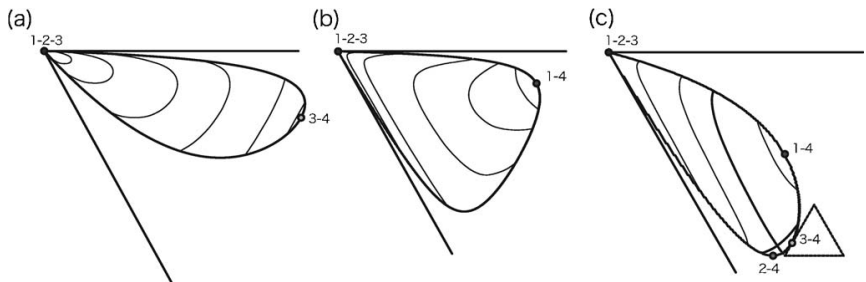

FIG. 12. The contour lines of the Hamiltonian for (a) $\Gamma_{1}=4, \Gamma_{2}=2$, $\Gamma_{3}=-1$, and $\Gamma_{4}=5$; (b) $\Gamma_{1}=4, \Gamma_{2}=2, \Gamma_{3}=-3$, and $\Gamma_{4}=3$; and (c) $\Gamma_{1}=4$, $\Gamma_{2}=2, \Gamma_{3}=-5$, and $\Gamma_{4}=1$.

\section{Case II: $\Gamma=2 \Gamma_{4}$}

It follows from (39)-(41) that we have

$l_{14}^{2}=\frac{\Gamma_{2} \Gamma_{3}}{\Gamma_{1} \Gamma_{4}} l_{23}^{2}+4, \quad l_{24}^{2}=\frac{\Gamma_{3} \Gamma_{1}}{\Gamma_{2} \Gamma_{4}} l_{31}^{2}+4, \quad l_{34}^{2}=\frac{\Gamma_{1} \Gamma_{2}}{\Gamma_{3} \Gamma_{4}} l_{12}^{2}+4$.

Hence, the condition for the existence of the fourth vortex point on the sphere is derived from

$$
-4 \leq \frac{\Gamma_{2} \Gamma_{3}}{\Gamma_{1} \Gamma_{4}} l_{23}^{2}, \quad \frac{\Gamma_{3} \Gamma_{1}}{\Gamma_{2} \Gamma_{4}} l_{31}^{2}, \quad \frac{\Gamma_{1} \Gamma_{2}}{\Gamma_{3} \Gamma_{4}} l_{12}^{2}<0 .
$$

With an argument similar to that in the previous subsection, we have only to deal with the case when $\Gamma_{1} \geq \Gamma_{2}>0>\Gamma_{3}$ and $\Gamma_{4}=\Gamma_{1}+\Gamma_{2}+\Gamma_{3}>0$.

The points of tangency for each $B_{i}=0$ are given by solving the equations $b_{1}+b_{2}+b_{3}=0,2 \Gamma_{1} \Gamma_{2} b_{1} b_{2}+2 \Gamma_{2} \Gamma_{3} b_{2} b_{3}$ $+2 \Gamma_{3} \Gamma_{1} b_{3} b_{1}-\left(\Gamma_{1} b_{1}\right)^{2}-\left(\Gamma_{2} b_{2}\right)^{2}-\left(\Gamma_{3} b_{3}\right)^{2}-b_{1} b_{2} b_{3}=0, \quad$ and $B_{i}=0$. They are represented by

$$
\begin{array}{r}
\left(b_{1}, b_{2}, b_{3}\right) \\
=\left(-4 \Gamma_{1} \Gamma_{4}, \frac{4 \Gamma_{1} \Gamma_{4}\left(\Gamma_{3}+\Gamma_{1}\right)}{2 \Gamma_{1}+\Gamma_{2}+\Gamma_{3}}, \frac{4 \Gamma_{1} \Gamma_{4}\left(\Gamma_{2}+\Gamma_{1}\right)}{2 \Gamma_{1}+\Gamma_{2}+\Gamma_{3}}\right), \\
\left(b_{1}, b_{2}, b_{3}\right) \\
=\left(\frac{4 \Gamma_{2} \Gamma_{4}\left(\Gamma_{3}+\Gamma_{2}\right)}{\Gamma_{1}+2 \Gamma_{2}+\Gamma_{3}},-4 \Gamma_{2} \Gamma_{4}, \frac{4 \Gamma_{2} \Gamma_{4}\left(\Gamma_{1}+\Gamma_{2}\right)}{\Gamma_{1}+2 \Gamma_{2}+\Gamma_{3}}\right), \\
\left(b_{1}, b_{2}, b_{3}\right) \\
=\left(\frac{4 \Gamma_{3} \Gamma_{4}\left(\Gamma_{2}+\Gamma_{3}\right)}{\Gamma_{1}+\Gamma_{2}+2 \Gamma_{3}}, \frac{4 \Gamma_{3} \Gamma_{4}\left(\Gamma_{1}+\Gamma_{3}\right)}{\Gamma_{1}+\Gamma_{2}+2 \Gamma_{3}},-4 \Gamma_{3} \Gamma_{4}\right) .
\end{array}
$$

The points (43) and (45) coincide with $b_{2}=0$ when $\Gamma_{3}+\Gamma_{1}=0$, and the points (43) and (44) agree with $b_{3}=0$ when $\Gamma_{3}+\Gamma_{2}=0$. Therefore, the distribution of the points of tangency changes when $\Gamma_{3}=-\Gamma_{1}$ or $\Gamma_{3}=-\Gamma_{2}$. Thus, we show the contour plots of the Hamiltonian for the following three cases: $0>\Gamma_{3}>-\Gamma_{2},-\Gamma_{2}>\Gamma_{3}>-\Gamma_{1}$, and $-\Gamma_{2}>\Gamma_{3}>-\Gamma_{1}$ $-\Gamma_{2}$.

Figure 12(a) shows the contour lines for $\Gamma_{1}=4, \Gamma_{2}=2$, $\Gamma_{3}=-1$, and $\Gamma_{4}=5$. The point of tangency $3-4$ is elliptic and the structure of the contours is simple. Second, we show the contour plot of the Hamiltonian in Fig. 12(b) for $\Gamma_{1}=4$, $\Gamma_{2}=2, \Gamma_{3}=-3$, and $\Gamma_{4}=3$, which gives a topological structure similar to the first case except for the points of tangency 1-4. Third, the contour lines of the Hamiltonian for $\Gamma_{1}=4$, $\Gamma_{2}=2, \Gamma_{3}=-5$, and $\Gamma_{4}=1$ in Fig. 12(c) indicate that there are 
elliptic points of tangency $1-4,2-4$, and $3-4$, and one hyperbolic fixed point with homoclinic connections.

Finally, we note that the self-similar collapse of the vortex triple 123 cannot be ruled out in this case. Indeed, $\Gamma_{1}=4, \Gamma_{2}=2, \Gamma_{3}=-\frac{4}{3}$, and $\Gamma_{4}=\frac{14}{3}$ satisfy the necessary conditions in (17). This is another example where the selfsimilar triple collapse possibly occurs.

\section{SUMMARY AND DISCUSSION}

We have investigated the integrable motion of the four vortex points on the sphere, when the moment of vorticity is zero. The article completes the study of the integrable vortex problem on the sphere together with the integrable threevortex problem ${ }^{3-5}$. As in the four-vortex problem in the unbounded plane, ${ }^{7}$ we have successfully applied a reduction method, from a four-vortex problem to a three-vortex problem, as proposed by Rott. ${ }^{8}$ The evolution is classified by plotting the level curves of the Hamiltonian in the trilinear coordinates. For the spherical integrable four-vortex problem, the zero total vorticity condition is unnecessary to guarantee the integrability. However, the vortex strengths are strongly restricted by the fact that the distances between the vortex points are bounded.

We have considered whether or not the self-similar collapse is possible. No existence of the four-vortex collapse has been proved mathematically. We gave the necessary conditions for the triple collapse and the binary collapse, and checked them in Secs. IV and V. The binary collapse is proved to be impossible as a result, while the possibility of the triple collapse is unable to be ruled out theoretically. We found some examples of the vortex strengths for which the self-similar triple collapse could be possible. The detailed study of these cases is a future direction.

Finally, we look for periodic orbits that induce the pseudo-Anosov map on the sphere; To do this, for given vortex strengths, we set the four vortex points along the equator so that they satisfy $P=Q=S=0$, with which we track the evolution numerically. When the relative distances between the four vortex points become the same as those of the initial configuration, we check if the periodic orbit defines the pA four-braids or not. Strictly speaking, the four vortex points do not necessarily return to their initial positions after one-period evolution, since the periodic orbit is described in terms of the relative configuration. However, introducing an appropriate change of coordinate system, we map the four vortex points to their initial position, and thus we can also define the four-braids on the sphere. We have examined all the examples given in this paper. Unfortunately, almost all the four-braids look periodic, but we find a few orbits that induce the pA map for $\Gamma_{1}=5, \Gamma_{2}=3, \Gamma_{3}=-4$, and $\Gamma_{4}=-5$, an example of which is shown in Fig. 13(a). For the sake of comparison, we give an orbit that induces the periodic fourbraids in Fig. 13(b). Between these two orbits, we also show the contour lines corresponding to them in the physical region. Since the initial configurations of these two orbits are very close, so are the energy levels. Nevertheless, the topological structures of the periodic orbits are quite different. It would be interesting to investigate how the topological struc-

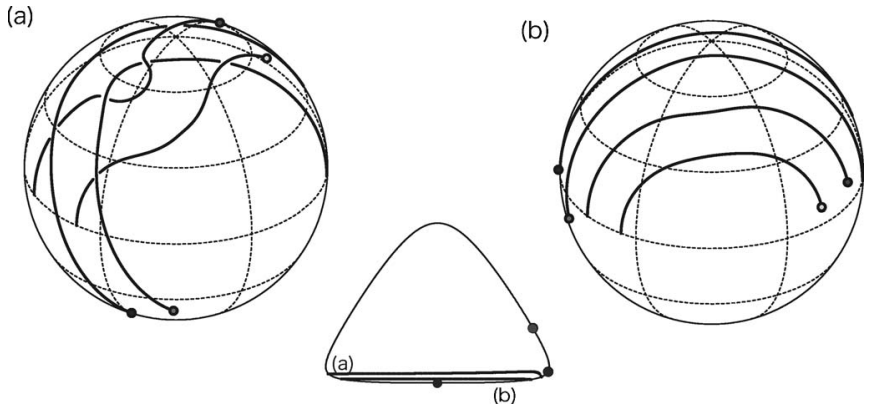

FIG. 13. Periodic orbits of the four vortex points for $\Gamma_{1}=5, \Gamma_{2}=3, \Gamma_{3}=-4$, and $\Gamma_{4}=-5$ that induce (a) the pA 4-braids and (b) the periodic 4-braids. The corresponding contour lines in the trilinear phase space are also shown between them.

ture of the periodic orbit affects the statistical property of the particle mixing on the sphere in spite of the close energy level, which is another future direction.

\section{ACKNOWLEDGMENTS}

This work is partially supported by Ministry of Education, Science, Sports and Culture, Grant-in-Aid for Young Scientists (A) No. 17684002 2006, Grant-in-Aid for Exploratory No. 17654018 2006, and Grant-in-Aid for formation of $\mathrm{COE}$ at Hokkaido University. I would like to thank Prof. Eiko Kin for useful discussions.

\section{APPENDIX: DEDUCTION OF THE UPPER BOUND FOR $L_{1}-L_{4}$}

The possible regions for the vortex strengths are derived just from the lower bounds $\min L_{i}<L_{i}$. Thus, we need to show that they are sufficient to ensure the upper bounds $L_{i}<\max L_{i}$. In this appendix, we give a detailed proof of this fact for the first case when $\Gamma_{1} \geq \Gamma_{2} \geq \Gamma_{3} \geq \Gamma_{4}>0$ and $\Gamma_{1}-\Gamma_{2} \leq \Gamma_{3}$. As for the other cases, we leave out the proof, since it is quite similar.

The possible region for $\Gamma_{1}-\Gamma_{2} \leq \Gamma_{2}$ in Fig. 2(a) is expressed in the following two ways:

$$
\begin{aligned}
& 0<\Gamma_{4} \leq \frac{1}{2}\left(\Gamma_{1}-\Gamma_{2}\right), \quad \Gamma_{1}-\Gamma_{2}-\Gamma_{4}<\Gamma_{3} \leq \Gamma_{2}, \\
& \frac{1}{2}\left(\Gamma_{1}-\Gamma_{2}\right)<\Gamma_{4} \leq \Gamma_{2}, \quad \Gamma_{4} \leq \Gamma_{3} \leq \Gamma_{2},
\end{aligned}
$$

or

$$
\frac{1}{2}\left(\Gamma_{1}-\Gamma_{2}\right)<\Gamma_{3} \leq \Gamma_{1}-\Gamma_{2}, \quad \Gamma_{1}-\Gamma_{2}-\Gamma_{3}<\Gamma_{4} \leq \Gamma_{3},
$$

$$
\Gamma_{1}-\Gamma_{2}<\Gamma_{3} \leq \Gamma_{2}, \quad 0<\Gamma_{4} \leq \Gamma_{3} .
$$

We then need to show that if the vortex strengths are in the possible region, the invariants $L_{i}$ satisfy the following upper bounds:

$$
\begin{aligned}
L_{1} & =\left(\Gamma_{2}+\Gamma_{3}+\Gamma_{4}\right)^{2}-\Gamma_{1}^{2}<\max L_{1} \\
& =4 \Gamma_{2} \Gamma_{3}+4 \Gamma_{2} \Gamma_{4}+4 \Gamma_{3} \Gamma_{4},
\end{aligned}
$$




$$
\begin{aligned}
L_{2} & =\left(\Gamma_{1}+\Gamma_{3}+\Gamma_{4}\right)^{2}-\Gamma_{2}^{2}<\max L_{2} \\
& =4 \Gamma_{1} \Gamma_{3}+4 \Gamma_{1} \Gamma_{4}+4 \Gamma_{3} \Gamma_{4}, \\
L_{3} & =\left(\Gamma_{1}+\Gamma_{2}+\Gamma_{4}\right)^{2}-\Gamma_{3}^{2}<\max L_{3} \\
& =4 \Gamma_{1} \Gamma_{2}+4 \Gamma_{1} \Gamma_{4}+4 \Gamma_{2} \Gamma_{4}, \\
L_{4} & =\left(\Gamma_{1}+\Gamma_{2}+\Gamma_{3}\right)^{2}-\Gamma_{4}^{2}<\max L_{4} \\
& =4 \Gamma_{1} \Gamma_{2}+4 \Gamma_{2} \Gamma_{3}+4 \Gamma_{3} \Gamma_{1} .
\end{aligned}
$$

First we show that (A1) implies (A5). Comparing $4 \Gamma_{2} \Gamma_{4}$ $+\Gamma_{1}^{2}$ with the square of $2 \Gamma_{2}+2 \Gamma_{4}-\Gamma_{1}$, we have

$$
\begin{aligned}
& \left(4 \Gamma_{2} \Gamma_{4}+\Gamma_{1}^{2}\right)-\left(2 \Gamma_{2}+2 \Gamma_{4}-\Gamma_{1}\right)^{2} \\
& \quad=4 \Gamma_{4}\left(\Gamma_{1}-\Gamma_{2}-\Gamma_{4}\right)+4 \Gamma_{2}\left(\Gamma_{1}-\Gamma_{2}\right) \geq 0,
\end{aligned}
$$

since $\Gamma_{1} \geq \Gamma_{2}$ and $\Gamma_{1}-\Gamma_{2}-\Gamma_{4} \geq \frac{1}{2}\left(\Gamma_{1}-\Gamma_{2}\right) \geq 0$ due to (A1). Hence, it follows from $\Gamma_{1}^{2}+4 \Gamma_{2} \Gamma_{4}>0$ and $2 \Gamma_{2}+2 \Gamma_{4}-\Gamma_{1}$ $\geq 2 \Gamma_{2}-\Gamma_{1}>0$ that

$$
\sqrt{\Gamma_{1}^{2}+4 \Gamma_{2} \Gamma_{4}} \geq 2 \Gamma_{2}+2 \Gamma_{4}-\Gamma_{1}>0 .
$$

Thus, we have $\Gamma_{2}+\Gamma_{4}-\sqrt{\Gamma_{1}^{2}+4 \Gamma_{2} \Gamma_{4}} \leq \Gamma_{1}-\Gamma_{2}-\Gamma_{4}<\Gamma_{3}$, due to (A1), which results in

$$
\sqrt{\Gamma_{1}^{2}+4 \Gamma_{2} \Gamma_{4}}>\Gamma_{2}-\Gamma_{3}+\Gamma_{4}>0 .
$$

On the other hand, when the vortex strengths satisfy (A2), we have $\Gamma_{2}+\Gamma_{4}-\sqrt{4 \Gamma_{2} \Gamma_{4}+\Gamma_{1}^{2}}<\Gamma_{4} \leq \Gamma_{3}$, since

$$
\begin{gathered}
4 \Gamma_{2} \Gamma_{4}+\Gamma_{1}^{2}-\Gamma_{2}^{2}>2 \Gamma_{2}\left(\Gamma_{1}-\Gamma_{2}\right)+\Gamma_{1}^{2}-\Gamma_{2}^{2} \\
\quad=\left(\Gamma_{1}+3 \Gamma_{2}\right)\left(\Gamma_{1}-\Gamma_{2}\right) \geq 0 .
\end{gathered}
$$

Hence, we have (A9) again. When both sides of (A9) are squared, we obtain

$$
\left(\Gamma_{2}+\Gamma_{3}+\Gamma_{4}\right)^{2}-\Gamma_{1}^{2}<4 \Gamma_{2} \Gamma_{3}+4 \Gamma_{2} \Gamma_{4}+4 \Gamma_{3} \Gamma_{4} .
$$

Second, we have the comparisons $4 \Gamma_{1} \Gamma_{4}+\Gamma_{2}^{2}-\left(2 \Gamma_{4}\right.$ $\left.+\Gamma_{2}\right)^{2}=4 \Gamma_{4}\left(\Gamma_{1}-\Gamma_{2}-\Gamma_{4}\right) \geq 0$ for (A1) and $4 \Gamma_{1} \Gamma_{4}+\Gamma_{2}^{2}-\Gamma_{1}^{2}$ $>2 \Gamma_{1}\left(\Gamma_{1}-\Gamma_{2}\right)+\Gamma_{2}^{2}-\Gamma_{1}^{2}=\left(\Gamma_{1}-\Gamma_{2}\right)^{2} \geq 0$ for (A2). Each of them leads to $\Gamma_{4}+\Gamma_{1}-\sqrt{4 \Gamma_{1} \Gamma_{4}+\Gamma_{2}^{2}} \leq \Gamma_{1}-\Gamma_{2}-\Gamma_{4}<\Gamma_{3}$, and $\Gamma_{1}+\Gamma_{4}-\sqrt{4 \Gamma_{1} \Gamma_{4}+\Gamma_{2}^{2}}<\Gamma_{4} \leq \Gamma_{3}$. Hence, we have $\sqrt{4 \Gamma_{1} \Gamma_{4}+\Gamma_{2}^{2}}>\Gamma_{1}-\Gamma_{3}+\Gamma_{4}>0$. Taking square of both sides, we have (A6).

Third, we use the expressions (A3) and (A4) to prove (A7). Since (A3) implies $4 \Gamma_{1} \Gamma_{2}+\Gamma_{3}^{2}-\left(2 \Gamma_{2}+\Gamma_{3}\right)^{2}=4 \Gamma_{2}\left(\Gamma_{1}\right.$
$\left.-\Gamma_{2}-\Gamma_{3}\right) \geq 0$, we have $\Gamma_{1}+\Gamma_{2}-\sqrt{4 \Gamma_{1} \Gamma_{2}+\Gamma_{3}^{2}} \leq \Gamma_{1}-\Gamma_{2}-\Gamma_{3}$ $<\Gamma_{4}$. As for (A4), it follows from $4 \Gamma_{1} \Gamma_{2}+\Gamma_{3}^{2}-\left(\Gamma_{1}+\Gamma_{2}\right)^{2}$ $=\left(\Gamma_{3}-\Gamma_{1}+\Gamma_{2}\right)\left(\Gamma_{3}+\Gamma_{1}-\Gamma_{2}\right)>0$, that we have $\Gamma_{1}+\Gamma_{2}$ $-\sqrt{\Gamma_{3}^{2}+4 \Gamma_{1} \Gamma_{2}}<0<\Gamma_{4}$. Hence, we acquire $\sqrt{4 \Gamma_{1} \Gamma_{2}+\Gamma_{3}^{2}}$ $>\Gamma_{1}+\Gamma_{2}-\Gamma_{4}>0$, from which the upper bound of $L_{3}$ is obtained.

Finally, we have $4 \Gamma_{1} \Gamma_{2}+\Gamma_{4}^{2}-\left(\Gamma_{4}+2 \Gamma_{2}\right)^{2}=4 \Gamma_{2}\left(\Gamma_{1}-\Gamma_{2}\right.$ $\left.-\Gamma_{4}\right) \geq 0$ due to (A1) and

$$
\begin{aligned}
4 \Gamma_{1} & \Gamma_{2}+\Gamma_{4}^{2}-\left(\Gamma_{1}+\Gamma_{2}-\Gamma_{4}\right)^{2}=2 \Gamma_{1} \Gamma_{2}+2 \Gamma_{1} \Gamma_{4}+2 \Gamma_{2} \Gamma_{4} \\
& -\Gamma_{1}^{2}-\Gamma_{2}^{2}>2 \Gamma_{1} \Gamma_{2}+\left(\Gamma_{1}+\Gamma_{2}\right)\left(\Gamma_{1}-\Gamma_{2}\right)-\Gamma_{1}^{2}-\Gamma_{2}^{2} \\
& =2 \Gamma_{2}\left(\Gamma_{1}-\Gamma_{2}\right) \geq 0,
\end{aligned}
$$

due to (A2), from which we obtain $\Gamma_{1}+\Gamma_{2}-\sqrt{\Gamma_{4}^{2}+4 \Gamma_{1} \Gamma_{2}}$ $\leq \Gamma_{1}-\Gamma_{2}-\Gamma_{4}<\Gamma_{3}$ for (A1) and $\Gamma_{1}+\Gamma_{2}-\sqrt{\Gamma_{4}^{2}+4 \Gamma_{1} \Gamma_{2}}<\Gamma_{4}$ $\leq \Gamma_{3}$ for (A2), respectively. Therefore, we have the inequality $0<\Gamma_{1}+\Gamma_{2}-\Gamma_{3}<\sqrt{\Gamma_{4}^{2}+4 \Gamma_{1} \Gamma_{2}}$, which yields (A8).

${ }^{1}$ Y. Kimura and H. Okamoto, "Vortex motion on a sphere," J. Phys. Soc. Jpn. 56, 4203 (1987).

${ }^{2} \mathrm{P}$. K. Newton, The N-vortex Problem, Analytical Techniques (Springer, New York, 2001).

${ }^{3}$ R. Kidambi and P. K. Newton, "Motion of three point vortices on a sphere," Physica D 116, 143 (1998).

${ }^{4}$ R. Kidambi and P. K. Newton, "Collision of three vortices on a sphere," Nuovo Cimento Soc. Ital. Fis., C 22C, 779 (1999).

${ }^{5}$ T. Sakajo, "The motion of three point vortices on a sphere," Jpn. J. Ind. Appl. Math. 16, 321 (1999).

${ }^{6}$ B. Eckhardt, "Integrable four vortex motion," Phys. Fluids 31, 2796 (1988).

${ }^{7}$ H. Aref and M. S. Stremler, "Four-vortex motion with zero total circulation and impulse," Phys. Fluids 11, 3704 (1999).

${ }^{8}$ N. Rott, "Constrained three- and four-vortex problems," Phys. Fluids A 2, 1477 (1990).

${ }^{9}$ P. Boyland, M. Stremler, and H. Aref, "Topological fluid mechanics of point vortex motions," Physica D 175, 69 (2003).

${ }^{10} \mathrm{~W}$. Thurston, "On the geometry and dynamics of diffeomorphism of surfaces," Bull., New Ser., Am. Math. Soc. 19, 417 (1988).

${ }^{11}$ P. Boyland, H. Aref, and M. Stremler, "Topological fluid mechanics of stirring," J. Fluid Mech. 403, 277 (2000).

${ }^{12} \mathrm{~J}$. Birman, "Braids, links, and mapping class groups," in Annals of Mathematical Studies Vol. 82 (Princeton University Press, Princeton, NJ, 1974).

${ }^{13}$ K. Murasugi and B. Kurpita, "A study of braids," Mathematics and its Applications Vol. 484, Kluwer Academic, Dordrecht, 1999).

${ }^{14}$ Y. Kimura, "Chaos and collapse of point vortices," Fluid Dyn. Res. 3, 98 (1988).

${ }^{15}$ H. Aref, "Motion of three vortices," Phys. Fluids 22, 393 (1979). 